\title{
Stress correlations in granular materials: An entropic formulation
}

\author{
G. Lois, ${ }_{1}^{1,2}$ J. Zhang, ${ }^{3}$ T. S. Majmudar, ${ }^{3}$ S. Henkes, ${ }^{4}$ B. Chakraborty ${ }^{4}$ C. S. O’Hern, ${ }^{1,2}$ and R. P. Behringer ${ }^{3}$ \\ ${ }^{1}$ Department of Mechanical Engineering, Yale University, New Haven, Connecticut 06520-8284, USA \\ ${ }^{2}$ Department of Physics, Yale University, New Haven, Connecticut 06520-8120, USA \\ ${ }^{3}$ Department of Physics, Duke University, P.O. Box 90305, Durham, North Carolina 27708, USA \\ ${ }^{4}$ Department of Physics, Brandeis University, Waltham, Massachusetts 02454, USA
}

(Received 29 May 2009; revised manuscript received 12 October 2009; published 28 December 2009)

\begin{abstract}
We study the response of dry granular materials to external stress using experiment, simulation, and theory. We derive a Ginzburg-Landau functional that enforces mechanical stability and positivity of contact forces. In this framework, the elastic moduli depend only on the applied stress. A combination of this feature and the positivity constraint leads to stress correlations whose shape and magnitude are extremely sensitive to the nature of the applied stress. The predictions from the theory describe the stress correlations for both simulations and experiments semiquantitatively.
\end{abstract}

DOI: 10.1103/PhysRevE.80.060303

A striking feature of dry granular materials and other athermal systems is that they form force chain networks in response to applied stress, with large forces distributed inhomogeneously into linear chainlike structures $[1,2]$. A number of experimental studies have visualized and quantified these networks in granular systems using carbon paper [3] and photoelastic techniques $[4,5]$. These studies demonstrated that geometrical and mechanical properties of force chain networks are acutely sensitive to preparation procedures, especially near the jamming transition [6]. For example, in isotropically compressed systems, force networks are ramified with only short-ranged spatial correlations of the stress. In contrast, in sheared systems, aligned force chains give rise to longer-ranged stress correlations in the compressive direction.

Developing theoretical descriptions for the mechanical properties of granular media is challenging for several important reasons [7-10]: (1) since tensile stresses are absent in dry granular materials they only remain intact via applied stress, making the limiting zero-stress isostatic state-where the number of degrees of freedom matches the number of constraints [11-13] — problematic; (2) forces at the microscopic level are indeterminate due to friction and disorder; (3) granular materials are athermal, so that conventional energy-based statistical approaches are not appropriate; and (4) near isostaticity we expect fluctuations to be important, both within a single realization of a system and from realization to realization. New methods are needed to bridge the gap between force networks at small length scales and continuum elastoplastic theory at large scales, and to capture the highly sensitive, fluctuating behavior of granular systems near jamming.

In this Rapid Communication, we construct a model for stress fluctuations based on grain-scale force and torque balance, positivity of contact forces, and entropy maximization. We then calculate stress correlations and predict differences for systems under isotropic compression versus pure shear. We also perform complementary numerical simulations and experimental studies of jammed granular systems in twodimensions (2D) subject to isotropic compression and pure shear. The stress correlation functions from theory, simulation, and experiment are in qualitative and in some cases quantitative agreement. The theory predicts that the form of
PACS number(s): 45.70.-n, 64.60.-i, 83.80.Fg

the stress correlations depends on how jammed packings were prepared. In particular, for sheared systems, real-space stress correlations decay more quickly in the expanded compared to the compressed direction. Further, the simulations show that this result is only weakly dependent on friction.

Theoretical framework. Fluctuations are related to the number of microscopic states available under a given set of macroscopic conditions. In equilibrium thermodynamics, the microcanonical entropy determines fluctuations and response. However, classifying states according to energy is not useful for granular media, and a different classification scheme is needed. The approach that we pursue exploits a different conservation principal-one based on force and torque balance, which applies rigorously for granular materials [14-16].

The force-moment tensor of mechanically stable (MS) packings, $\hat{\Sigma}=\int d^{d} r \hat{\sigma}(\mathbf{r})$, where $\hat{\sigma}(\mathbf{r})$ is the local stress tensor, is an extensive variable and topological invariant $[14,17]$. In the force-moment ensemble, $\hat{\Sigma}$ remains fixed barring systemspanning changes. Hence, local fluctuations only involve grain configurations with the same $\hat{\Sigma}$. To construct a theory for stress correlations, we adopt a coarse-grained approach, in which jammed configurations are represented by a continuous field [18] and the entropy $S(\hat{\Sigma})$ is defined via an appropriate Ginzburg-Landau functional. The theory is valid close to jamming where grains have negligible deformations, and stress fluctuations decouple from volume fluctuations [19].

In two and three dimensions, a continuous field can be defined that upholds force and torque balance $[17,20]$ of granular packings. We focus on 2D systems, where a scalar field $\Psi$, the Airy stress function [21], is related to the local stress tensor by

$$
\hat{\sigma}(\mathbf{r})=\left[\begin{array}{cc}
\partial_{y}^{2} \Psi & -\partial_{x} \partial_{y} \Psi \\
-\partial_{x} \partial_{y} \Psi & \partial_{x}^{2} \Psi
\end{array}\right] .
$$

We define $\Gamma=\operatorname{Tr} \hat{\Sigma}$ and $\tau=s_{1}-s_{2}=\sqrt{\Gamma^{2}-4(\operatorname{det} \hat{\Sigma})^{2}}$, where $s_{1}$ $>s_{2}$ are eigenvalues of $\hat{\Sigma}$. Given $\hat{\Sigma}, \Psi$ can be expanded as $\Psi_{0}+\psi$, where $\psi$ gives the fluctuations around $\Psi_{0}, \Psi_{0}$ satis- 
fies the biharmonic equation, $\nabla^{4} \Psi_{0}=0$ [21], and $\Sigma_{i j}$ $=\int d^{d} r\left(\delta_{i j} \nabla^{2} \Psi_{0}-\partial_{i} \partial_{j} \Psi_{0}\right)[17]$.

The probability for fluctuations is given by $P[\psi]$ $=Z^{-1}(\hat{\Sigma}) e^{-\mathrm{L}\left[\Psi_{0}, \psi\right]} \equiv Z^{-1}(\hat{\Sigma}) e^{-\mathrm{L} \hat{\Sigma}[\psi]}$, where the functional $\mathrm{L}_{\hat{\Sigma}}[\psi]$ measures the entropy from grain packings that have coarsegrained representation $\psi(\mathbf{r})$. The partition function $Z(\hat{\Sigma})$ $\equiv e^{S(\hat{\Sigma})}=\int D \psi e^{-\mathrm{L} \hat{\Sigma}[\psi]}$ generates correlators of the field $\psi[18]$. Due to gauge freedom, terms in $L_{\hat{\Sigma}}[\psi]$ can only depend on second and higher derivatives of $\psi$. The coefficients of these terms and, therefore, the strength of fluctuations are controlled by $\Psi_{0}$ (or $\left.\hat{\Sigma}\right)$. To lowest order in $\psi$ and its derivatives, $\mathrm{L}_{\hat{\Sigma}}[\psi]$ resembles the free energy for a $2 \mathrm{D}$ elastic material [21],

$$
\begin{aligned}
\mathrm{L}_{\hat{\Sigma}}[\psi]= & \int d^{2} r\left\{\alpha_{1}(\hat{\Sigma})\left(\partial_{x}^{2} \psi\right)^{2}+\alpha_{2}(\hat{\Sigma})\left(\partial_{y}^{2} \psi\right)^{2}\right. \\
& \left.+\alpha_{3}(\hat{\Sigma})\left(\partial_{x}^{2} \psi\right)\left(\partial_{y}^{2} \psi\right)+\cdots\right\} .
\end{aligned}
$$

Differences between Eq. (2) and traditional elasticity theory are (a) the stiffness constants are not intrinsic material properties but are determined by $\hat{\Sigma}$ and (b) the origin of the functional is entropic, not energetic with the stiffness constants related to the entropy $S(\hat{\Sigma})[17]$.

There are many MS packings at fixed $\hat{\Sigma}$, each characterized by a given $\psi(\mathbf{r})$. Pressure correlations are determined by averaging over these configurations. If configurations are sampled according to $P[\psi] \propto e^{-\mathrm{L} \hat{\Sigma}[\psi]}$, correlations in simulations and experiments should be well described by the fieldtheoretic predictions. Below, we use Eq. (2) to calculate pressure correlations for packings under isotropic compression and pure shear.

Isotropic compression. For this case, the stiffness constants only depend on $\Gamma$ and $L_{\hat{\Sigma}}[\psi]$ is isotropic,

$$
\begin{aligned}
\mathrm{L}_{\Gamma}[\psi]= & \int d^{2} r\left[\frac{K(\Gamma)}{2}\left\{\left(\partial_{x}^{2} \psi\right)^{2}+\left(\partial_{y}^{2} \psi\right)^{2}+2 \partial_{x}^{2} \psi \partial_{y}^{2} \psi\right\}\right. \\
& \left.+K^{\prime \prime} \xi^{\prime 2}\left(\nabla^{3} \psi\right)^{2}\right]
\end{aligned}
$$

with stiffness coefficient $K(\Gamma)$, a higher-order gradient term with a $\hat{\Sigma}$-independent coefficient $K^{\prime \prime} \xi^{\prime 2}$, and length scale $\xi^{\prime}$ associated with grain-scale variations of $\psi$.

Positivity of contact forces implies that $\partial_{x}^{2} \Psi$ and $\partial_{y}^{2} \Psi$ are non-negative $[14,17]$. Positivity of the long-wavelength fluctuations can be enforced in a mean-field way by requiring that $K(\Gamma) \geq 1 / \Gamma^{2}[17,22]$. For frictionless packings, which are isostatic at jamming [23], an exact calculation yields $K(\Gamma)=e^{-S[\Gamma]}=z_{\text {iso }} /\left(2 \Gamma^{2}\right)[17]$, where the isostatic contact number $z_{\text {iso }}=4$ in two dimensions. In contrast, $z_{\text {iso }}=3$ for frictional disks, and frictional packings are typically hyperstatic with $z>z_{\text {iso }}$ [24]. We do not have an exact result for $K(\Gamma)$ away from isostaticity; however, simulations can be used to obtain $K(\Gamma)$. We have measured $S(\Gamma)$ in packings of frictionless disks under isotropic compression [17] and found a good fit to the form $K(\Gamma)=\left[z_{\text {iso }} / 2+c\left(z-z_{\text {iso }}\right)^{2}\right] / \Gamma^{2}$, with $c \simeq 2$. We use this form for $K(\Gamma)$ to compare predictions for the pressure correlations with those from simulations and experi- (a)
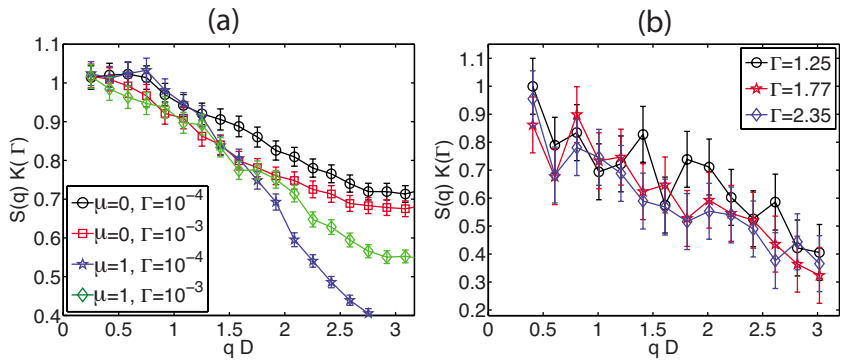

FIG. 1. (Color online) Angle-averaged stress correlations $S(q) K(\Gamma)$ for isotropic compression from (a) simulations at different applied pressures $\Gamma$ (reduced units) and static friction coefficients $\mu$ and (b) experiments at $\mu=0.7$ and different $\Gamma$ (units of $\mathrm{N} \mathrm{m}$ ). Theory predicts $S(q) K(\Gamma) \approx 1$ independent of $\Gamma$ and $q$ for $q \ll 1 / \xi$ $\approx 1 / D$.

ments for frictionless and frictional packings with appropri-

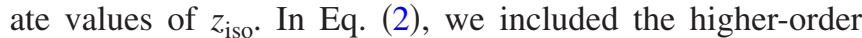
derivative term proportional to $\left(\nabla^{3} \psi\right)^{2}$ to facilitate comparisons between theory, experiments, and simulations, where the grain scale is small but not negligible compared to the system size. We verify a posteriori that $\xi=\xi^{\prime} \sqrt{K^{\prime \prime} / K(\Gamma)}$ is on the order of a grain diameter and independent of $\Gamma$.

The results for local pressure correlations are easily visualized in Fourier space. From Eq. (3), these correlations are isotropic,

$$
S(\mathbf{q})=\left\langle|\delta \Gamma(\mathbf{q})|^{2}\right\rangle=q^{4}\left\langle|\psi(\mathbf{q})|^{2}\right\rangle=\frac{K^{-1}(\Gamma)}{1+\xi^{2} q^{2}},
$$

where $\mathbf{q}$ is the wave vector. Thus, we predict under isotropic compression that $S(\mathbf{q}) K(\Gamma) \rightarrow 1$ for $q \ll 1 / \xi$ independent of $\Gamma$.

To test predictions for q-space pressure fluctuations, we performed simulations and experiments on 2D packings. We numerically generated MS packings of bidisperse disks $(N / 2$ large and $N / 2$ small particles with a diameter ratio $r=1.4$ ) with and without friction near jamming using well-known algorithms $[25,26]$. The simulations allowed us to investigate the role of friction in determining stress correlations. We studied system sizes ranging from $N=256$ to 4096, square cells and periodic boundary conditions, pressures in the range $\Gamma / A=10^{-5}-10^{-3}$ (in units of the grain stiffness), and static friction coefficients $\mu=[0,1]$. We have also carried out experiments using a biaxial apparatus described previously $[6,27,28]$. The biax is a device that allows us to apply highly controlled deformations to systems of photoelastic disks, where it is possible to measure all contacts and contact forces. In this study, contact forces are calculated for $N$ $=1228$ disks, with $N / 5$ large and $4 N / 5$ small disks, $r=1.2$, and $\mu=0.7$.

In isotropically compressed systems, pressure fluctuations $S(\mathbf{q})$ decay isotropically. Thus, in Fig. 1 we plot the angleaveraged $S(q)$, multiplied by $K(\Gamma)$, from simulations [Fig. $1(\mathrm{a})$ and experiments [Fig. 1(b)] versus $q D$, where $D$ is the average particle diameter. For frictional and frictionless grains over a wide range of $\Gamma$, the simulation and experimental results match Eq. (4) at $q \ll 1 / D$ with no fitting parameters. The simulations and experiments confirm the predicted 
(a)

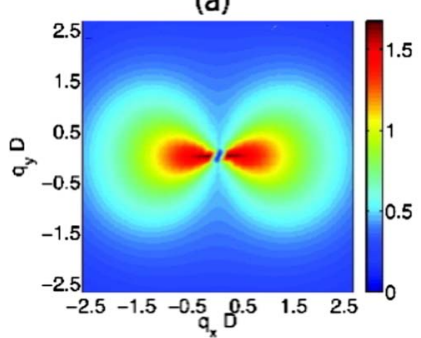

(c)

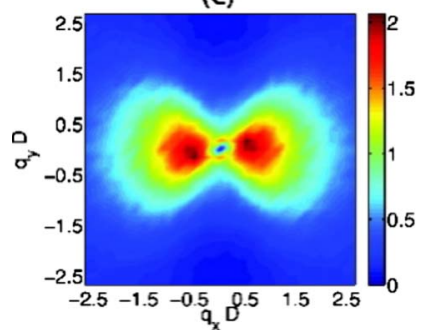

(b)

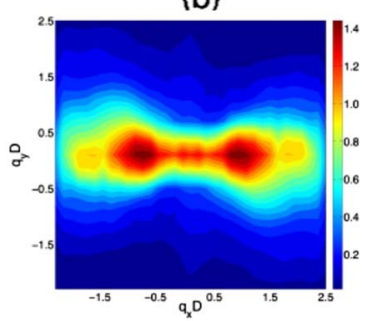

(d)

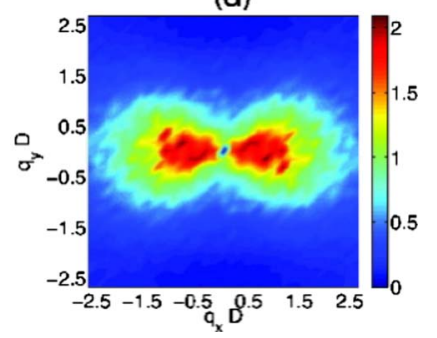

FIG. 2. (Color online) Contours of $S(q) K(\Gamma)$ under pure shear from (a) theory $(\tau / \Gamma=0.3)$, (b) experiment $(\tau / \Gamma=0.51)$, and simulations of (c) frictionless and (d) frictional $(\mu=1)$ particles $(\tau / \Gamma$ $=0.3$ ). In all panels, compression (dilation) is along the vertical (horizontal) axis.

scaling of $S(q) \sim 1 / K(\Gamma)$ and increasing stiffness as the system is decompressed.

Pure shear. For pure shear, the stress positivity constraint leads to different conditions on the stiffness constant $K$ in the $x$ and $y$ directions. A dramatic consequence is that $S(\mathbf{q})$ is anisotropic even for infinitesimal shear. We postulate a generalization of the entropic form for $K$ under isotropic compression and write

$$
\begin{aligned}
\mathrm{L}_{\tau, \Gamma}[\psi]= & \int d^{2} r\left[\frac{K(\Gamma+\tau)}{2}\left(\partial_{x}^{2} \psi\right)^{2}+\frac{K(\Gamma-\tau)}{2}\left(\partial_{y}^{2} \psi\right)^{2}\right. \\
& \left.+2 K^{\prime}(\Gamma, \tau) \partial_{x}^{2} \psi \partial_{y}^{2} \psi+K^{\prime \prime} \xi^{\prime 2}\left(\nabla^{3} \psi\right)^{2}\right] .
\end{aligned}
$$

Here, $x(y)$ is the principal axis of $\hat{\Sigma}$ with the smaller (larger) eigenvalue, and two distinct stiffness coefficients ensure that $\hat{\sigma}_{x x}$ and $\hat{\sigma}_{y y}>0$. In addition, $K^{\prime}$ controls fluctuations that

contribute to $\hat{\sigma}_{x x} \hat{\sigma}_{y y}$. We have also included the higher-order derivative term as in the isotropic case. The pressure correlations predicted from Eq. (5) are

$$
\begin{aligned}
S(\mathbf{q}) K(\Gamma)= & q^{4} K(\Gamma) /\left\{K(\Gamma+\tau) q_{x}^{4}+K(\Gamma-\tau) q_{y}^{4}+2 K^{\prime} q_{x}^{2} q_{y}^{2}\right. \\
& \left.+K^{\prime \prime} \xi^{\prime 2} q^{6}\right\} .
\end{aligned}
$$

Note that the positivity constraint does not impose any conditions on $K^{\prime}$ and $K^{\prime \prime}$, which are therefore taken to be independent of $\Gamma$ and $\tau$ near jamming where the $\Gamma$ dependence of the stiffness constant $K$ dominates. A crucial feature of Eq. (6) is that the pressure correlations are anisotropic, i.e., $\lim _{q_{\mathrm{y}} \rightarrow 0} S\left(0, q_{y}\right) \neq \lim _{q_{x} \rightarrow 0} S\left(q_{x}, 0\right)$.

To compare theory with experiment, we create a sheared packing by first isotropically compressing the system to a MS packing slightly above jamming. We then apply pure shear by expanding the system in one direction while compressing in the other at a fixed density. The resultant pressure correlations are presented in Fig. 2(b), and they match the expected anisotropic dipolar features predicted by Eq. (6) and shown in Fig. 2(a). To compare theory and simulation, we generated MS packings with and without friction over a range of stress ratios $\tau / \Gamma$. To do this, we compressed (dilated) the simulation cell in the $y(x)$ direction by $\epsilon=\delta L / L$ over the range $\epsilon=\left[10^{-5}, 10^{-3}\right]$. Pressure correlations from simulations of frictionless [Fig. 2(c)] and frictional [Fig. 2(d)] particles also show a strong dipolar signature.

A key prediction of Eq. (6) is that $\lim _{q \rightarrow 0} S(\mathbf{q})$ depends on direction. This feature is clearly demonstrated in Fig. 3, which shows the simulation and experimental results for $S(\mathbf{q}) K(\Gamma)$ along different cuts and predictions from theory. There is semiquantitative agreement between theory and simulations without friction for $q_{x}=0$ and $q_{y}=0$ cuts [Fig. $3(\mathrm{a})$ ]. We find similar results for simulations of frictional grains. Even though our theory makes several simplifying assumptions $\left(z-z_{\text {iso }} \ll 1\right.$ and $\left.\tau / \Gamma \ll 1\right)$, we also observe qualitative agreement between theory and experiments. In simulations and experiments, $q$-space pressure correlations depend on the approach to $q=0$, and they are larger along the expanded direction, as predicted by theory. Note that the peak in $S(\mathbf{q})$ in the expanded direction at $q_{x}>0$ is a finitesize effect as demonstrated in the insets, and thus we expect even better agreement for larger systems.

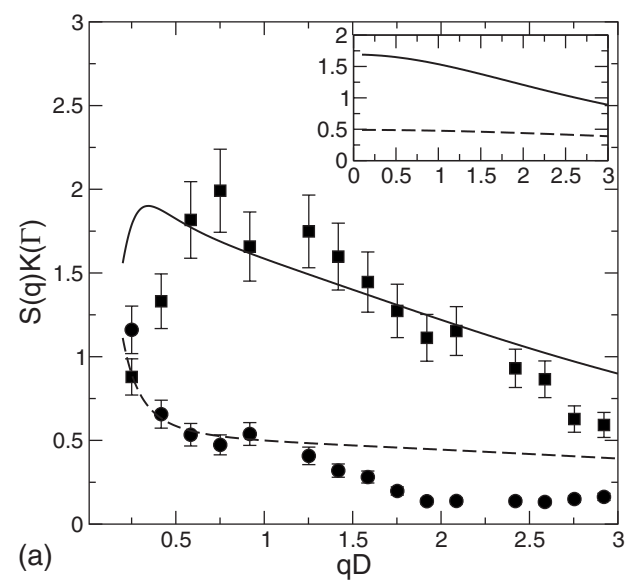

(b)

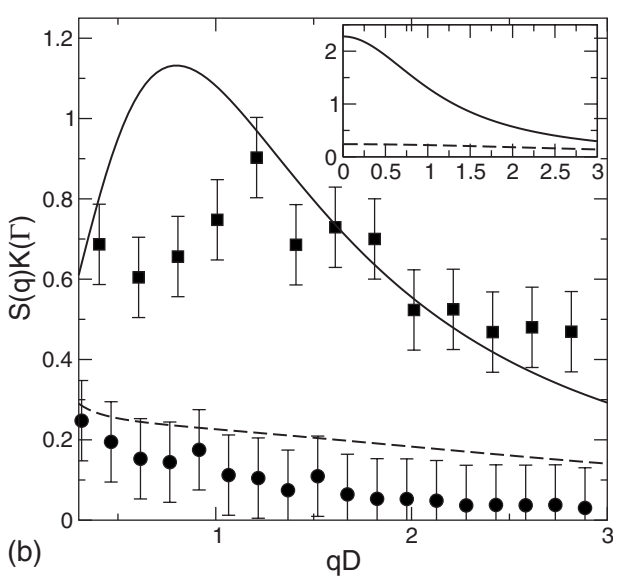

FIG. 3. Cuts in $S(q) K(\Gamma)$ along expanded (squares and solid lines) and compressed (circles and dashed lines) directions for (a) simulations at $\tau / \Gamma=0.3$ and $\mu=0$ and (b) experiments at $\tau / \Gamma=0.51$ and $\mu=0.7$ compared to theoretical predictions (lines). To obtain predictions in the main panels, finite low- $q$ cutoffs were used in Eq. (6), $\xi \sim D$ was obtained by fitting Eq. (4) to the isotropic data in Fig. 1, and $K^{\prime} / K(\Gamma) \sim O(1)$ was adjusted to best match the sheared data. The insets show the theoretical results for an infinite system. 

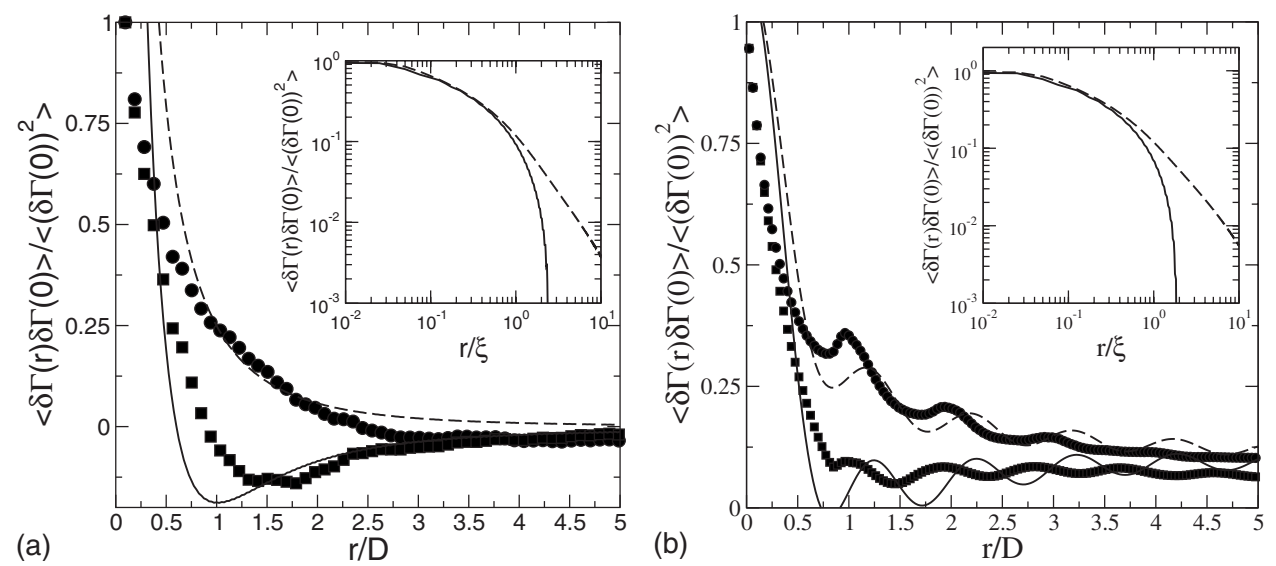

(b)
FIG. 4. Decay of pressure correlations $\langle\delta \Gamma(r) \delta \Gamma(0)\rangle /\left\langle[\delta \Gamma(0)]^{2}\right\rangle$ along compressed (circles and dashed lines) and expanded (squares and solid lines) directions under pure shear for the same systems in Fig. 3. Theoretical predictions (lines), which have been scaled by an overall constant to match correlations in the expanded direction at 0.5 , are compared to (a) simulations and (b) experiments (symbols). The insets show the infinite system results.
The $q$-space correlations for pure shear imply anisotropic decays in real space with a slower decay along the compressive direction. In Fig. 4, we present real-space pressure correlations $\langle\delta \Gamma(r) \delta \Gamma(0)\rangle /\left\langle[\delta \Gamma(0)]^{2}\right\rangle$ and find qualitative agreement between theory, simulations, and experiments. Thus, we argue that the entropic formulation with the positivity constraint provides an explanation for shear-induced anisotropy in pressure correlations observed in experiments [6].

We presented a field-theoretic approach for describing pressure fluctuations in granular systems. The theory enforces mechanical stability and uses load-dependent stiffness constants derived from the entropy of packings. The theory ensures positivity of contact forces and applies close to the jamming transition. Friction plays no special role. From the theory, we calculate pressure correlations and show that they depend sensitively on the packing preparation. Under isotropic compression, correlations are isotropic and obey a simple scaling relation with compression. For pure shear, correlations are anisotropic with dipolar features in $q$ space. The anisotropy is a consequence of the positivity constraint, which causes $q$-space pressure fluctuations to be reduced along the compressive direction. This feature in $q$ space gives rise to longer-ranged real-space pressure correlations in the compressive direction. Our approach provides a means to relate stress fluctuations to the history of granular systems, which determines the force-moment tensor, and an explanation for the anisotropic behavior of pressure fluctuations. The theoretical predictions for the pressure correlation functions are confirmed, semiquantitatively, by simulations of MS packings with and without friction and by experiments on photoelastic disks. This agreement provides evidence that the entropy of MS packings can be used to determine the response of granular and other nonequilibrium systems.

This work was supported by NSF Grants No. DMR0555431 (B.C. and S.H.), No. DMR0448838 (C.S.O. and G.L.), and No. DMR0555431 (J.Z., T.S.M., and R.P.B.), and by YINQE (G.L.). B.C. acknowledges discussions with Nick Read, and B.C., C.S.O., and G.L. recognize the Aspen Center for Physics and Lorentz Center, where aspects of this work were performed.
[1] C.-h. Liu et al., Science 269, 513 (1995).

[2] J. Zhou et al., Science 312, 1631 (2006).

[3] D. M. Mueth, H. M. Jaeger, and S. R. Nagel, Phys. Rev. E 57, 3164 (1998).

[4] P. Dantu, Ann. Ponts Chaussees 4, 144 (1967).

[5] J. Geng, et al., Phys. Rev. Lett. 87, 035506 (2001).

[6] T. S. Majmudar and R. P. Behringer, Nature (London) 435, 1079 (2005).

[7] M. Otto et al., Phys. Rev. E 67, 031302 (2003).

[8] M. E. Cates et al., Phys. Rev. Lett. 81, 1841 (1998).

[9] R. Blumenfeld, Phys. Rev. Lett. 93, 108301 (2004).

[10] I. Goldhirsch and C. Goldenberg, Eur. Phys. J. E 9, 245 (2002).

[11] A. V. Tkachenko and T. A. Witten, Phys. Rev. E 60, 687 (1999).

[12] C. F. Moukarzel, Phys. Rev. Lett. 81, 1634 (1998).

[13] M. Wyart, S. R. Nagel, and T. A. Witten, EPL 72, 486 (2005).

[14] S. Henkes, C. S. O’Hern, and B. Chakraborty, Phys. Rev. Lett. 99, 038002 (2007).

[15] R. Blumenfeld, in Granular and Complex Materials, Lecture Notes in Complex Systems Vol. 8, edited by T. Aste, A. Tordessilas, and T. D. Matteo (World Scientific, Singapore, 2007).
[16] B. P. Tighe, A. R. T. van Eerd, and T. J. H. Vlugt, Phys. Rev. Lett. 100, 238001 (2008).

[17] S. Henkes and B. Chakraborty, Phys. Rev. E 79, 061301 (2009).

[18] N. Goldenfeld, Lectures on Phase Transitions and the Renormalization Group (Addison-Wesley, New York, 1992).

[19] C. Song, P. Wang, and H. A. Makse, Nature (London) 453, 629 (2008).

[20] R. C. Ball and R. Blumenfeld, Phys. Rev. Lett. 88, 115505 (2002).

[21] L. D. Landau and E. M. Lifshitz, Theory of Elasticity (Butterworth-Heinemann, London, 1986).

[22] B. Tighe (private communication).

[23] C. S. O'Hern et al., Phys. Rev. E 68, 011306 (2003).

[24] L. E. Silbert, et al., Phys. Rev. E 65, 031304 (2002).

[25] N. Xu, J. Blawzdziewicz, and C. S. O’Hern, Phys. Rev. E 71, 061306 (2005).

[26] H. P. Zhang and H. A. Makse, Phys. Rev. E 72, 011301 (2005).

[27] T. S. Majmudar et al., Phys. Rev. Lett. 98, 058001 (2007).

[28] J. Zhang et al. (unpublished). 